\title{
Frequency and temperature dependence of the relative permittivity in ferroelectrics: Monte-Carlo simulation study
}

\author{
D. Bolten, ${ }^{\text {a) }}$ U. Böttger, and R. Waser \\ Institut für Werkstoffe der Elektrotechnik, University of Technology RWTH Aachen, D-52056 Aachen, \\ Germany
}

(Received 16 September 2002; accepted 18 December 2002)

\begin{abstract}
In this article, the domain wall densities calculated from simulated domain configurations are used to estimate the domain wall contributions to the dielectric permittivity of a ferroelectric material. The configurations were calculated using a Monte-Carlo model proposed by B. G. Potter et al. [J. Appl. Phys. 87, 4415 (2000)]. The temperature and frequency dependence of the contributions are analyzed and compared to experimental results obtained on $\mathrm{PbZr}_{x} \mathrm{Ti}_{1-x} \mathrm{O}_{3}$ thin films. (C) 2003 American Institute of Physics. [DOI: 10.1063/1.1544649]
\end{abstract}

\section{INTRODUCTION}

It has long been known that the dielectric permittivity $\varepsilon$ of a ferroelectric increases considerably during switching by an external electric field (square pulse form). ${ }^{1-4} \mathrm{~A}$ similar increase is also observed when the switching field varies slowly with time in a sinusoidal fashion (e.g., during the measurement of the small-signal capacitance, $C-V$ curve). This increase in permittivity is traditionally attributed to domain wall contributions in addition to the intrinsic lattice response. The domain wall contributions change when the domain wall configuration is changed by applying a bias field and a typical double-peak " $C-V$ " curve results. While numerous experimental and theoretical work has been devoted to the study of the domain wall contributions to the dielectric and piezoelectric properties of ferroelectric materials,${ }^{5-11}$ only few efforts have been made to model the small-signal permittivity as a function of external field. ${ }^{12}$

According to the accepted theory, the domain wall contributions are directly proportional to the domain wall area present in the material. This quantity is almost impossible to extract from experimental data and only the volume fractions of, for example, $90^{\circ}$ or $180^{\circ}$ domains can be obtained from $\mathrm{x}$-ray diffraction experiments. ${ }^{13}$ Monte-Carlo simulation provides a reliable way to estimate this quantity since the simulation calculates the domain configurations for each point of a hysteresis curve. This data can then be analyzed regarding the domain wall area of the different domain wall types present. By assigning extrinsic contributions to each domain wall type, the domain wall contributions to the permittivity can be calculated and compared with experiment.

\section{EXPERIMENTAL METHODOLOGY}

\section{A. Model description}

The two-dimensional (2-D) Monte-Carlo simulation used to calculate the domain patterns was based on a model published by Potter $e t$ al. ${ }^{14}$ The model takes into account the dipole-dipole interaction, the gradient energy, and the energy of the dipoles in an external field. Details on the model

${ }^{a)}$ Electronic mail: bolten@iwe.rwth-aachen.de and its implementation can be found in Ref. 15 In order to extract the domain wall densities, an algorithm was implemented into the simulation program that decomposed the domain configuration into individual domains and subsequently classified the domain walls and calculated their length (for a 2-D simulation volume the domain wall area had the unit of a length.). The domain wall density was obtained by division with the simulation volume. The domains were classified into $c$-domains (i.e., the spontaneous polarization $P_{s}$ oriented parallel or antiparallel to the $y$-direction, the direction of the external electric field) and $a$-domains ( $P_{s}$ parallel or antiparallel to the $x$-direction). As a result, three different types of domain walls occurred: $180^{\circ}$ walls between adjacent $c$-domains, $90^{\circ}$ walls between $c$ - and $a$-domains and $180^{\circ}$ walls between adjacent $a$-domains. The domain wall configurations calculated during a hysteresis simulation were processed in this way to obtain the domain wall densities as a function of external field. An example domain configuration of a $100 \times 100$ lattice is shown in Fig. 1. The simulation started with a completely random configuration that was allowed to evolve for 400 Monte-Carlo steps per spin (MCS/ spin). Free boundary conditions were employed.

\section{B. Experimental procedure}

To compare the simulation results with experimental data, $\mathrm{PbZr}_{x} \mathrm{Ti}_{1-x} \mathrm{O}_{3}$ films (PZT) of composition 30/70 and $45 / 55$ were prepared. The PZT films were deposited by a 2-butoxyethanol-based chemical solution deposition spin coating process. The butoxyethanol-based precursor solution was synthesized by a modified method. ${ }^{16,17}$ The films were deposited on standard commercial platinized Si-wafers $\left[\mathrm{Si} / \mathrm{SiO}_{2} / \mathrm{TiO}_{2} / \mathrm{Pt}(100 \mathrm{~nm})\right]$ from aixACCT Laboratories, Aachen, Germany. After each layer deposition, the films were pyrolized at 200 and $400^{\circ} \mathrm{C}$ in air for 2 min each. The thickness of the film was varied by the number of deposition/ pyrolysis cycles. After depositing the last layer the final crystallization was performed at $700{ }^{\circ} \mathrm{C}$ in $\mathrm{O}_{2}$ with a rapid thermal annealing process. Pt top electrodes of $100 \mathrm{~nm}$ were sputtered at room temperature and structured with a photolithography/lift-off process. A post-anneal was performed at $700^{\circ} \mathrm{C}$ in $\mathrm{O}_{2}$ to recover sputter-induced damages. 


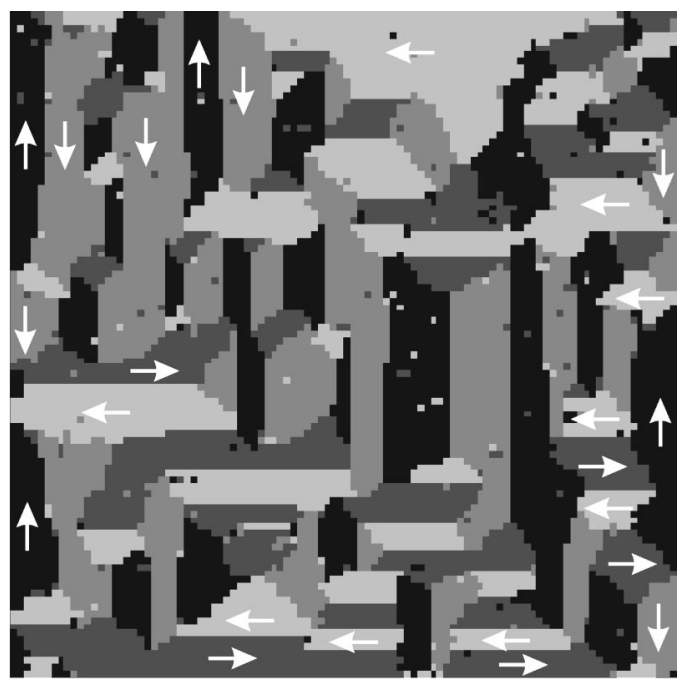

Legend

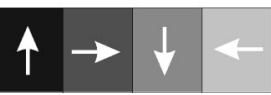

FIG. 1. Domain configuration of a $100 \times 100$ lattice after $400 \mathrm{MCS} / \mathrm{spin}$.

Most hysteresis curves presented in this work were measured with the aixACCT TF analyzer 2000, a computer based measurement tool to characterize ferroelectric thin films. The signal generation, amplification, A/D conversion, calculation, and graphical representation were all performed with a desktop computer. The system allowed hysteresis measurements in the dynamical range between $1 \mathrm{~Hz}$ to $1 \mathrm{kHz}$, with a maximal amplitude of $10 \mathrm{~V}$.

The capacitance-voltage dependence was measured with a HP 4284A LCR-bridge. The equipment used allowed the variation of the amplitude $U_{A C}$ of the small-signal measuring signal between $5 \mathrm{mV}$ up to $10 \mathrm{~V}$. By using the bias feature of the bridge, a dc voltage could be superimposed on the small measuring signal, thus allowing to measure the permittivity at different polarization states (which correspond to different points of the ferroelectric hysteresis). By changing the dc-bias in a step-like fashion, a complete $C-V$ curve was measured. Since this measurement was controlled via the IEEE488 bus with a computer, the dc-bias could only be changed slowly, resulting in a large-signal frequency of approximately $100 \mathrm{mHz}$.

For higher frequencies of the large signal, a different method had to be used. Here, the slowly varying large signal was generated with an arbitrary wave form generator (Wavetek 395). On this signal, a small-signal measuring voltage (the reference channel of a PerkinElmer DSP Model 7280 lock-in amplifier) was superimposed electronically and then fed to the sample. The answer of the sample was then applied to the input of the lock-in amplifier. With this setup, a dynamic range of the large signal excitation between a few $\mathrm{mHz}$ up to $10 \mathrm{~Hz}$ was possible.

\section{RESULTS AND DISCUSSION}

\section{A. Temperature dependence}

Figure 2 displays an example of the evolution of the domain densities for $180^{\circ}, 90^{\circ}$, and $180^{\circ}$ domain walls

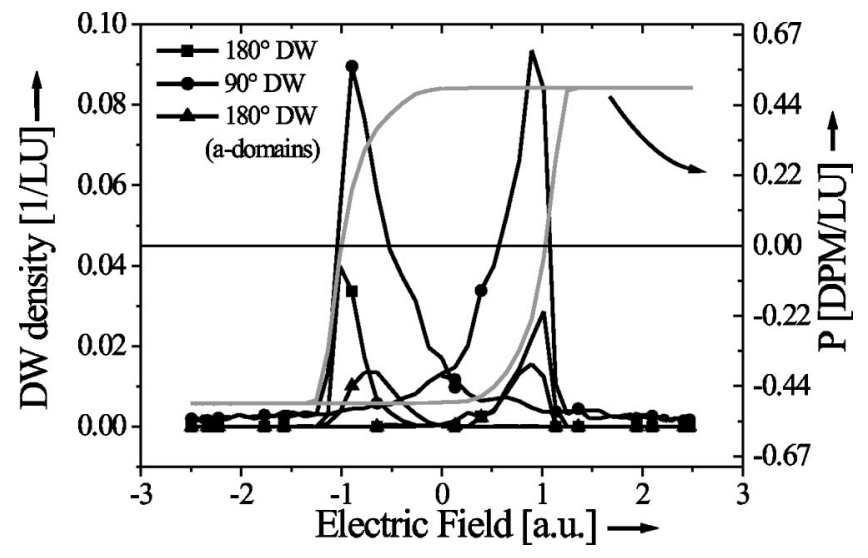

FIG. 2. Evolution of domain wall densities during a hysteresis simulation $\left(150 \times 150\right.$ lattice, $\left.k_{B} T=1\right)$.

(within $a$-domains) present in the simulation volume of a $150 \times 150$ lattice during a hysteresis simulation. The corresponding simulated hysteresis curve is also shown in the graph. The $180^{\circ}$ domain walls within $a$-domains (i.e., domains that were uniformly polarized parallel to the $x$-direction of the simulation volume) were treated separately from other $180^{\circ}$ domain walls because they did not contribute to the permittivity for an electric field applied in the $y$-direction (as will be later assumed), since no force was acting on them.

Interestingly, only the maxima of the "regular" $180^{\circ}$ domain wall density coincided with the coercive field of the hysteresis curve, while the $90^{\circ}$ and $180^{\circ}$ ( $a$-domains) domain wall concentrations reached their maxima before the coercive field was established. The usage of the free boundary condition implicitly penalized the simulation for building up polarization charges on the boundary of the simulation volume. ${ }^{14,15}$ As a consequence, the domains at the boundary had their polarization vector pointing mostly parallel to the boundary, as can be seen in Fig. 1. This implies that backswitching mainly occurs by $90^{\circ}$ switching processes at the point of remanent polarization. These $a$-domains can act as nucleation sites. When the external field was increased towards the coercive field, the $a$-domains started growing, while at the same time new c-domains with $\mathrm{P}$ parallel $\mathrm{E}$ nucleated. At first sight, it appears counterintuitive that the $a$-domains also grew since the parallel orientation had the lowest energy density. However, no nuclei for $a$-domains had to be formed since they were already present at the point of remanent polarization and their growth also reduced the total energy of the system. The increase of the $180^{\circ}$ ( $a$-domain) domain wall concentration and subsequently the increase of the $90^{\circ}$ concentration was due to the dipoledipole interaction. In this early switching state, the creation of $90^{\circ}$ domain walls reduced the number of situations with head-to-head or tail-to-tail configurations. Often a newly created $90^{\circ}$ wall also entailed the simultaneous creation of $180^{\circ}$ wall within an $a$-domain. Once the parallel c-domains had grown through the sample and expanded laterally, the number of $90^{\circ}$ walls and $180^{\circ}$ walls ( $a$-domains) started to decrease rapidly. 
This argument illustrates that the creation of $90^{\circ}$ walls can also be energetically favorable just due to electrostatic interactions alone. Whether such domain walls are actually created in real materials depends on the mechanical energies necessary to create $90^{\circ}$ domain walls and whether the energy gain is not consumed by the mechanical energies used for their creation.

To arrive at $\varepsilon-E$ curves, the domain wall contributions to the permittivity have to be taken into account. To this end, the following model is used. If an electric field is applied parallel to a $180^{\circ}$ wall that separates two antiparallel polarized regions, the domain wall will move as a whole a distance $x$ favoring the domain polarized parallel to the direction of the external field. Assuming that the domain wall moves in a harmonic potential $\frac{1}{2} k x^{2}$ with force constant $k$, the domain wall's deflection can be calculated when the pressure $2 P_{s} E$ is acting on the wall. With the relation $\mathrm{P} \approx D$ $=\varepsilon_{0} \varepsilon E$, the contributions to the permittivity by $180^{\circ}$ domain walls can be shown to be

$$
\varepsilon_{0} \Delta \varepsilon^{180^{\circ}} \approx \frac{4 P_{s}^{2} n_{D W}^{180^{\circ}}}{k_{180^{\circ}}},
$$

while for $90^{\circ}$ domain walls the corresponding contribution reads as

$$
\varepsilon_{0} \Delta \varepsilon^{90^{\circ}} \approx \frac{\sqrt{2} P_{s}^{2} n_{D W}^{90^{\circ}}}{2 k_{90^{\circ}}}
$$

where $n_{D W}^{180^{\circ}}, n_{D W}^{90^{\circ}}, k_{180^{\circ}}$, and $k_{90^{\circ}}$ denote the domain wall concentrations and force constants for $180^{\circ}$ and $90^{\circ}$ domain walls, respectively, and $P_{s}$ is the magnitude of the spontaneous polarization. The different numerical factors in these formulas reflect the different geometries.

Using these equations and the domain wall densities extracted from the domain configurations calculated during hysteresis simulations, the domain wall contributions to the permittivity were calculated. The resulting contributions for three different temperatures are depicted in Fig. 3. $\Delta \varepsilon$ was normalized with the spontaneous polarization $P_{s}$ to eliminate the dependence on $P_{s}$ from the calculated permittivity. A direct comparison of Monte-Carlo simulation data to experimental data is difficult due to the small simulation volume. Thus, only qualitative properties of the simulated dependence could be compared with experiment. It was further assumed that $k_{90^{\circ}}=k_{180^{\circ}}=1$. In reality, the force constants for $180^{\circ}$ and $90^{\circ}$ domain wall motion will surely be different. However, only few estimates of these constants have been published in the literature. Furthermore, to study the qualitative behavior, an assumption like the one made above appears to be valid. A drawback of the simulation approach used was that no temperature dependence of the spontaneous polarization was taken into account during the simulation. However, while the magnitude of the spontaneous polarization decreases with increasing temperature, the magnitude of the force constants decreases. By inspection of Eqs. (1) and (2) it becomes apparent that these changes will partially cancel each other, so that the overall qualitative behavior is approximately preserved.

Figure 4(a) displays the relative permittivity of a $30 / 70$

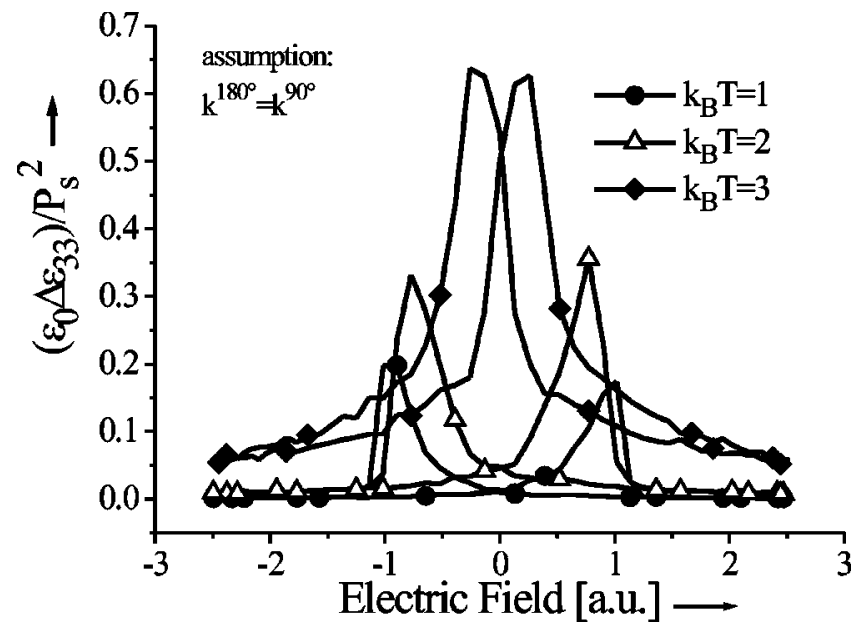

FIG. 3. Domain wall contributions to the permittivity as function of temperature. Calculated with Eqs. (1) and (2) from the domain wall densities extracted from hysteresis simulations.

PZT thin film of thickness $200 \mathrm{~nm}$ measured using the lock-in technique as a function of temperature $(f=1 \mathrm{~Hz}$, $\left.f_{A C}=10 \mathrm{kHz}, E_{A C}=5 \mathrm{kV} / \mathrm{cm}\right)$. Fig. $4(\mathrm{~b})$ shows the domain wall contributions to the permittivity derived from
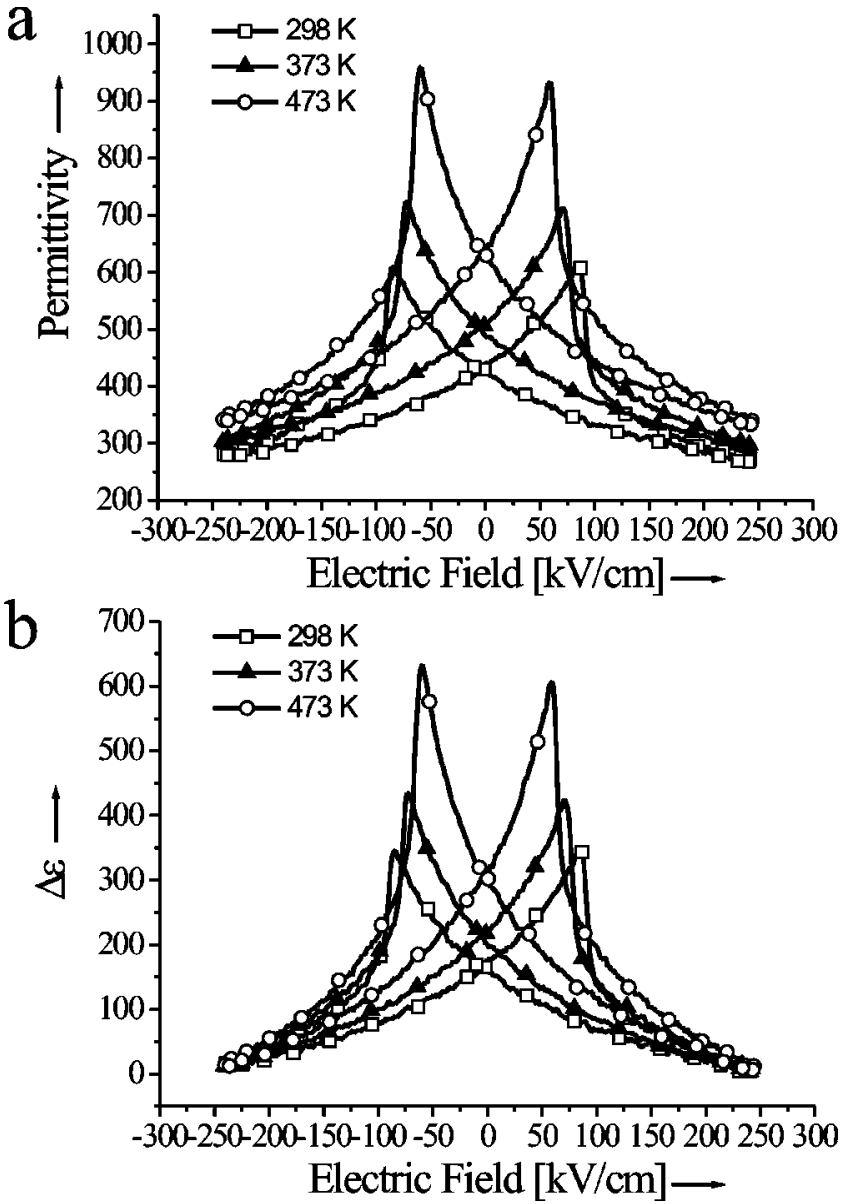

FIG. 4. (a) Relative permittivity of a 200-nm-thick 30/70 PZT thin film ( $f$ $=1 \mathrm{~Hz}, f_{A C}=10 \mathrm{kHz}, E_{A C}=5 \mathrm{kV} / \mathrm{cm}$ ). (b) Domain wall contributions to the permittivity. 
Fig. 4(a) by assuming that in the point of saturation (i.e., at maximal applied field) all domain walls are driven out of the sample and the intrinsic contributions dominate. With increasing temperature the maxima are shifted towards the $E$ $=0$ axis, while the maxima increase at the same time. The same trend can clearly be seen in Fig. 3, which shows the domain wall contributions of $180^{\circ}$ and $90^{\circ}$ walls to the permittivity derived from simulated domain wall densities.

The domain wall contributions to the permittivity displayed in Fig. 4(b) do not show any temperature dependence in the point of saturation (by construction). The relative permittivity [see Fig. 4(a)], however, still displays a temperature dependence due, of course, partly to the increase of the intrinsic permittivity as the phase transition temperature is approached. Figure 3, however, suggests that at higher temperatures, the permittivity at saturation is not completely due to intrinsic contributions. Thus, the common practice to estimate the intrinsic contributions of a ferroelectric at the point of saturation might not be reliable for high temperatures.

\section{B. Frequency dependence}

The frequency dependence of the simulated domain wall contributions is also fairly easily accessible with MonteCarlo simulations. The frequency dependence of the hysteresis curve was simulated by changing the number of MonteCarlo steps/spin that were performed at each field increment. ${ }^{14} \mathrm{~A}$ result of such a frequency variation is shown in Fig. 5(a) for a $150 \times 150$ lattice. The frequency is simply given by the reciprocal of the total simulation time (in units of MCS/spin). The highest frequency resulted for an equilibration time of $25 \mathrm{MCS} / \mathrm{spin}$ for each field increment (of 160 total), the lowest for an equilibration time of $300 \mathrm{MCS} / \mathrm{spin}$. The calculated hysteresis curves exhibited the typical broadening in field direction; that is, an increase in the coercive field. The simulated frequency dependence is very similar to the one observed experimentally. Subsequently, the domain wall concentrations were derived from the calculated domain configurations, as previously described, and used to estimate the domain wall contributions to the permittivity. The results are displayed in Fig. 5(b). As could be expected, the position of the maxima was shifted outwards with increasing frequency as a consequence of the increasing coercive field, but at the same time the peak height also increased.

While there is abundant experimental data on the ac frequency dependence of $C-V$ curves (i.e., the small-signal frequency dependence), virtually no information on the frequency effect of the slowly varying dc signal on the permittivity can be found in literature. ${ }^{18}$ Figure 6 displays the permittivity of a PZT 45/55 film measured for various frequencies of the slowly varying large signal (the measuring ac excitation was kept constant at $100 \mathrm{kHz}, 50 \mathrm{mV}$ ). The 1-mHz measurement was performed with a bridge, while all the others were recorded using the lock-in amplifier technique. The inset shows a close-up of the maxima. The experimental data is in complete agreement with the simulation results. For increasing frequency, the peak position was shifted to higher fields, while the peak height increased simultaneously.
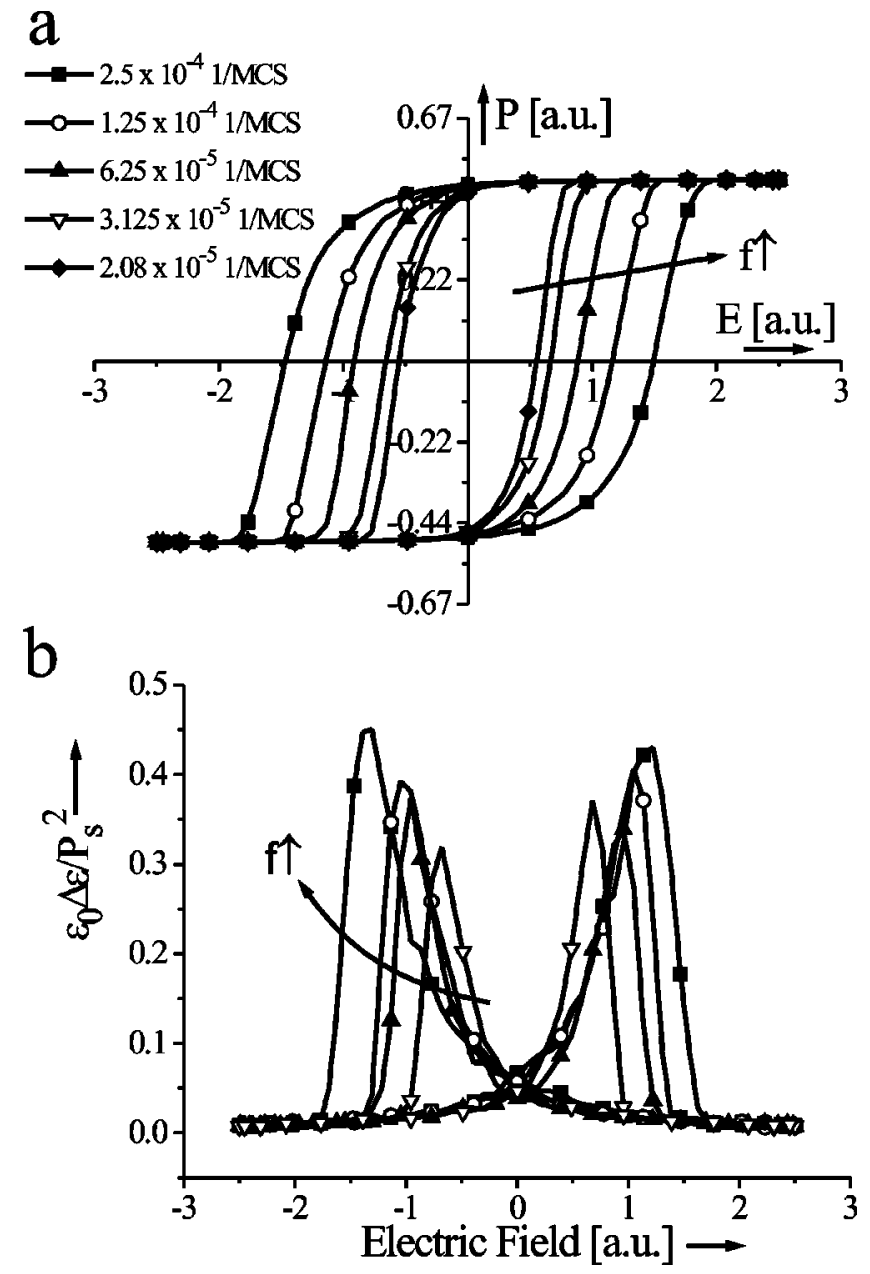

FIG. 5. (a) Frequency dependence of the simulated hysteresis curves. (b) Resulting frequency dependence of the derived domain wall contributions to the permittivity.

Unfortunately, the Monte-Carlo simulation technique does not allow the simulation of the dependence of the capacitance on the frequency of the small-signal excitation. The minute changes in domain wall configurations due to an

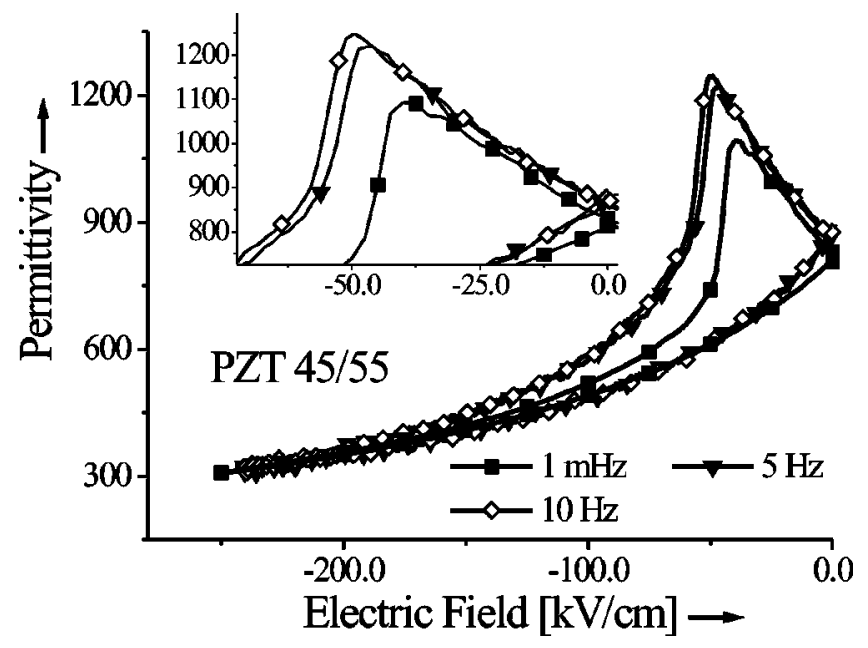

FIG. 6. $\varepsilon-E$ curves of a PZT 45/55 film measured at different frequencies of the dc bias. The inset displays a close-up of the maxima. 
ac signal with high frequency and small amplitude would be completely masked by the random nature of the simulation process itself. It does, however, provide a clue as to the possible cause. It was demonstrated that the side-wise motion of a domain wall proceeds by nucleation and lateral growth of kinks onto the existing wall in the used Monte-Carlo model. ${ }^{15}$ The simulation of the switching process showed that new nuclei are not created instantaneously but that this process needs time. Therefore, it appears plausible that the frequency dependence of the capacitance on the small-signal frequency is due to the nature of the nucleation process itself. For low ac frequencies, new kinks can readily form onto existing domain walls, giving a large domain wall contribution to the permittivity. As the ac frequency is increased, less time is available for the nucleation process and fewer kinks are formed, entailing a decreased domain wall contribution to the permittivity. Thus, the capacitance decreases with increasing ac frequency, as is observed experimentally. This model also explains why the frequency dependence is most pronounced in the vicinity of the coercive field and practically absent in saturation which is simply related to the maximal and minimal domain wall density at the coercive field and saturation, respectively.

\section{CONCLUSION}

In order to relate data on $C-V$ curves with domain wall densities that are difficult to obtain experimentally, MonteCarlo simulations were performed to provide insight into the nonlinear dielectric response of ferroelectrics. The simulations were used to extract domain wall concentrations in a 2-D lattice model. In conjunction with a theoretical model of the domain wall contributions to the permittivity, this information was used to calculate the domain wall contributions as a function of the external electric field. The simulated $C-V$ curves show the characteristic shape that is also observed experimentally, which provides strong evidence that the variation of capacitance with electric field is due mainly to domain wall contributions. The simulated frequency and temperature dependence of $C-V$ curves were also in good qualitative agreement with experiments performed on PZT thin films. Thus, this approach can be used successfully to model the permittivity-field dependence of a ferroelectric.

The simulation results also provided a natural explanation for the dependence of the small-signal capacitance on the exciting small-signal's frequency. Since the motion of domain walls in the simulation proceeds by nucleation and growth of new nuclei onto an existing wall, the dependence of the small-signal capacitance on frequency might be due to the time needed for new nucleation processes to occur, thereby limiting the domain wall contributions to the permittivity at high frequencies.

Monte-Carlo simulations proved to be a viable tool to develop further insight into the physics of ferroelectrics, and further research to incorporate interface effects and piezoelectric contributions into the current model is underway.

\footnotetext{
${ }^{1}$ M. Drougard, H. Funk, and D. Young, J. Appl. Phys. 25, 1166 (1954).

${ }^{2}$ E. Fatuzzo, J. Appl. Phys. 32, 1571 (1961).

${ }^{3}$ A. Fouskova and V. Janousek, J. Phys. Soc. Jpn. 20, 1619 (1965).

${ }^{4}$ A. Fouskova and V. Janousek, J. Phys. Soc. Jpn. 20, 1625 (1965).

${ }^{5}$ C. Kittel, Phys. Rev. 83, 458 (1951).

${ }^{6}$ J. Fousek and V. Janousek, Phys. Status Solidi 13, 195 (1966).

${ }^{7}$ G. Arlt, H. Dederichs, and R. Herbiet, Ferroelectrics 74, 37 (1987).

${ }^{8}$ G. Arlt and N. Pertsev, J. Appl. Phys. 70, 2283 (1991).

${ }^{9}$ Q. Zhang, H. Wang, N. Kim, and L. Cross, J. Appl. Phys. 75, 454 (1994).

${ }^{10}$ M. Stula, J. Fousek, H. Kabelka, M. Fally, and H. Warhanek, J. Korean Phys. Soc. 32, 758 (1998).

${ }^{11}$ S. Trolier-McKinstry, J. J. Shephard, J. Lacey, T. Su, G. Zavala, and J. Fendler, Ferroelectrics 206, 381 (1998).

${ }^{12}$ F. Chai, J. Brews, R. Schrimpf, and D. P. Birnie III, J. Appl. Phys. 82, 2505 (1997)

${ }^{13}$ L. Cheng-Cui, Z. Xiao-Wen, and X. Yi-Jiang, Ferroelectrics 37, 623 (1981).

${ }^{14}$ B. Potter, V. Tikare, and B. Tuttle, J. Appl. Phys. 87, 4415 (2000).

${ }^{15}$ D. Bolten, U. Böttger, and R. Waser, Jpn. J. Appl. Phys. 41, 7202 (2002).

${ }^{16}$ K. Budd, S. Dey, and D. Payne, Br. Ceram. Proc. 36, 107 (1985).

${ }^{17}$ R. Nouwen, J. Mullens, D. Franco, J. Yperman, and L. C. Poucke, Vib. Spectrosc. 10, 291 (1996).

${ }^{18}$ C. Cho, W. Lee, B. Yu, and B. Kim, J. Appl. Phys. 86, 2700 (1999).
} 